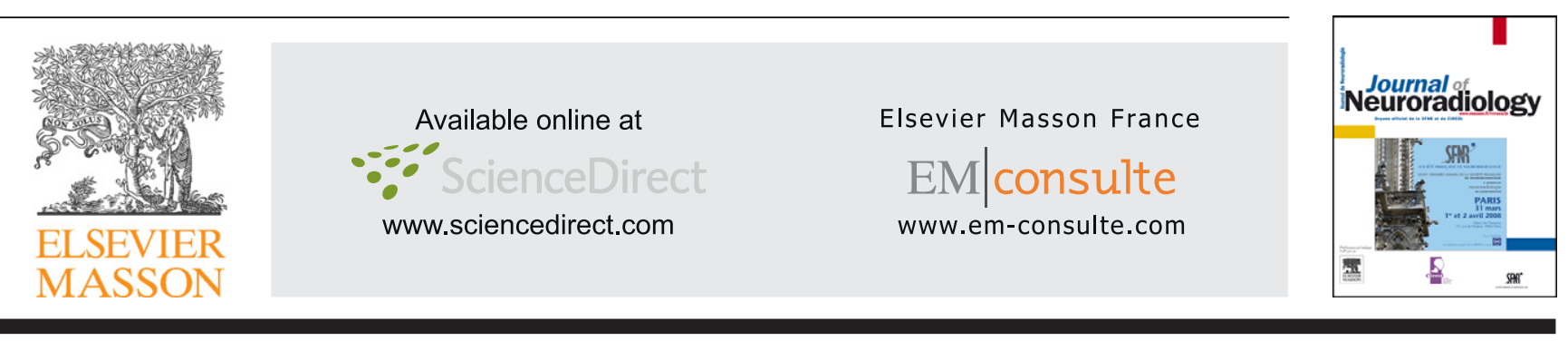

\title{
Epidermal nevus syndrome: An unusual cerebellar involvement
}

\section{Syndrome du naevus épidermique: une anomalie sous-tentorielle rare}

\author{
S. Pereira ${ }^{a, *}$, D. Serra ${ }^{b}$, P.M. Freitas ${ }^{a}$, B. Santiago ${ }^{c}$, O. Brito ${ }^{a}$
}

a Department of Imagiology-Neuroradiology, University Hospital of Coimbra, Praceta Prof Mota Pinto, 3000-075 Coimbra, Portugal

b Department of Dermatology, University Hospital of Coimbra, Praceta Prof Mota Pinto, 3000-075 Coimbra, Portugal

c Department of Neurology, University Hospital of Coimbra, Praceta Prof Mota Pinto, 3000-075 Coimbra, Portugal

Available online 13 August 2009

\section{KEYWORDS \\ Central nervous \\ system; \\ Cerebellum; \\ Congenital; \\ Epidermal nevus \\ syndrome}

\begin{abstract}
Summary The epidermal nevus syndrome is characterized by several developmental anomalies associated with an epidermal nevus. In addition to the skin, other organs commonly affected include the brain, eyes and musculoskeletal system. We report here on a 24-year-old woman with this syndrome who presented with hemifacial hypertrophy, hearing abnormalities, arrhythmia and an unusual infratentorial brain involvement.

(c) 2009 Elsevier Masson SAS. All rights reserved.
\end{abstract}

\section{Introduction}

The epidermal nevus syndrome (ENS) is a rare multisystemic syndrome. Patients present with an epidermal nevus, but are also affected by several developmental anomalies, most commonly of the brain, eyes and skeleton. Despite a growing body of knowledge, the full spectrum of the syndrome remains incompletely characterized.

Cerebellar involvement has been described, but always in association with supratentorial defects. However, we report here on a young adult woman who presented with ENS, but with an unusual cerebellar involvement. Magnetic resonance imaging (MRI) revealed enlargement of the left cerebellar hemisphere with disorganized folia and no supratentorial abnormalities.

\footnotetext{
* Corresponding author.

E-mail address: sara.leonor.pereira@gmail.com (S. Pereira).
}

\section{Case report}

Our 24-year-old female patient presented, since birth, a left-sided verrucous epidermal nevus that followed a linear blaschkoid course over her neck and face (Fig. 1). She also presented left-sided facial hypertrophy that was relatively mild at birth. She was the second child of nonconsanguineous healthy parents and her family history was negative.

During childhood, her psychomotor development was normal. However, when she started school, she was found to have learning difficulties. She underwent a special learning course and received psychological support.

At age 12, she had several near-syncope episodes. Physical examination disclosed mild difficulty in the foot-by-foot test, but no other coordination test abnormalities. The diagnostic work-up included neurological and cardiovascular evaluations. Cranial computed tomography (CT) revealed mild fourth ventricle dysmorphism, but no other changes, 


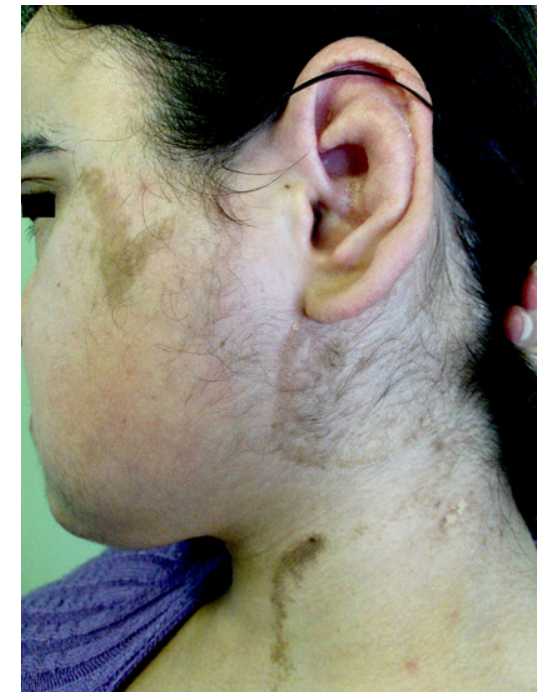

Figure 1 Left-sided cervicofacial verrucous epidermal nevus.

and the 24-h- and video-electroencephalography failed to detect any epileptogenic activity. However, her cardiac evaluation revealed paroxysmal supraventricular tachycardia caused by an atrioventricular bypass tract, which was successfully treated by catheter ablation.

As the patient grew older, her facial asymmetry (Fig. 2) continued to worsen, and she developed mechanical dysarthria due to hemilingual hypertrophy as well as ipsilateral neurosensory deafness. Several surgical procedures were performed to correct bony overgrowth, which required the implantation of metallic maxillary and mandibular prostheses.

\section{MRI findings}

At age 24, the metallic prostheses were removed to allow cranial MRI to be performed, using a 1.5-T unit and a standard head coil. T1- and T2-weighted images revealed diffuse cerebellar dysplasia, mainly of the left hemisphere and the vermis, with abnormal folia and fissure patterns,

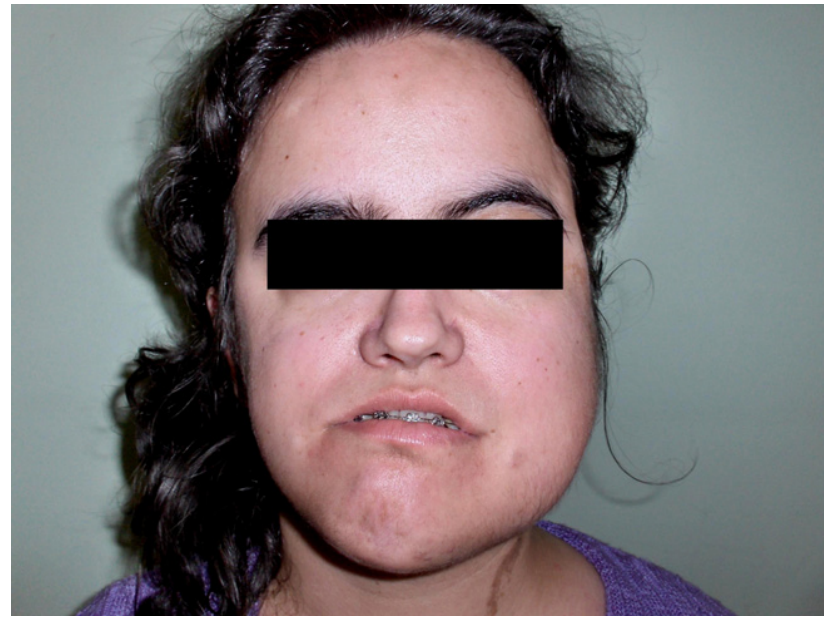

Figure 2 Left-sided facial hypertrophy.

and cortical polymicrogyria. Marked hypertrophy of the left hemisphere, foci of gray matter heterotopia, and effacement of the horizontal and superior fissures were also identified. There was an oblique orientation of the fissures in the superior portion of the left hemisphere, and a vertical orientation of the superior vermis fissures. The fourth ventricle was dysmorphic.

However, no supratentorial abnormalities were seen (Fig. 3). The images further documented previously known musculoskeletal changes-namely, hypertrophy of the leftsided cranial bones and contiguous soft tissues.

\section{Discussion}

In the present case, there are several features of the ENS-specifically, an epidermal nevus, hemifacial hypertrophy associated with mechanical dysarthria, left-sided neurosensory deafness, unusual structural brain changes, cognitive impairment and cardiac arrhythmia due to an abnormal AV bypass tract.

The ENS is a multisystemic syndrome that can present with a wide variety of signs and symptoms (Table 1) [1,2].
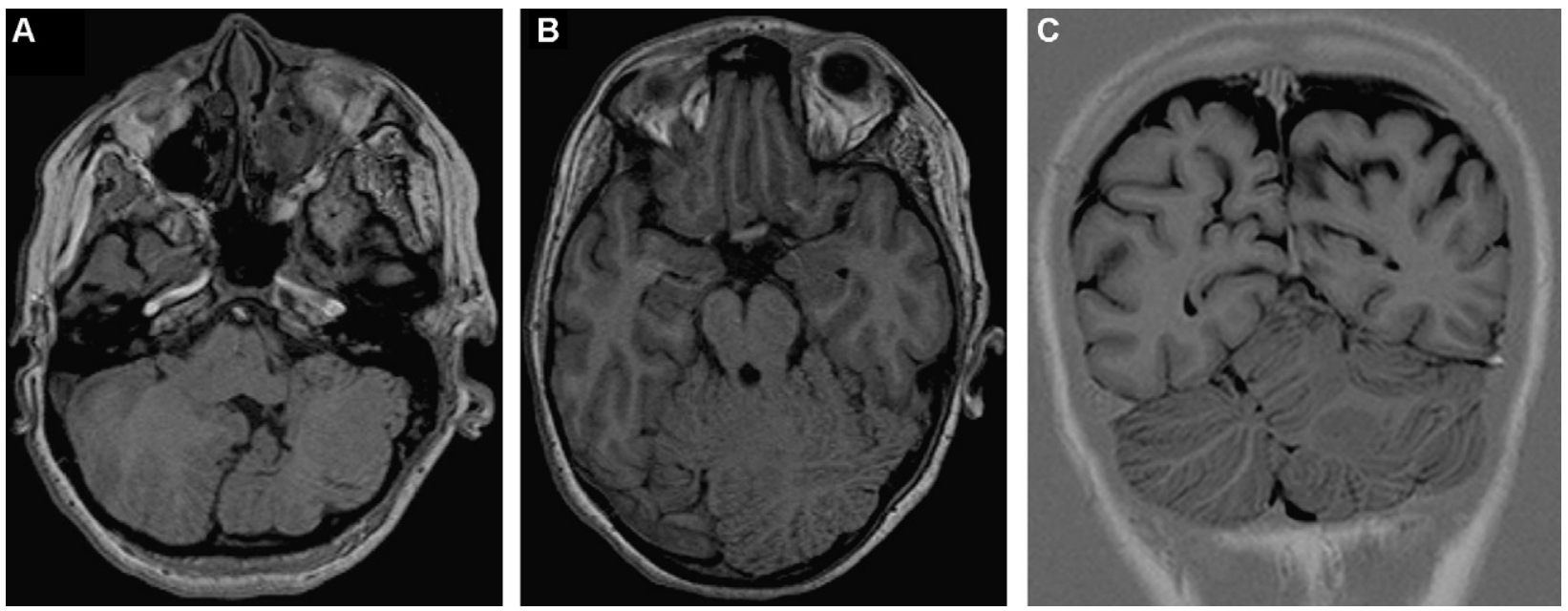

Figure 3 MRI revealing diffuse cerebellar dysplasia with left cerebellar hypertrophy. 
Table 1 The primary manifestations of the epidermal nevus syndrome (ENS).

\begin{tabular}{ll}
\hline Skin & Epidermal verrucous nevus \\
Musculoskeletal & Hemifacial hypertrophy \\
& Limb hypertrophy \\
Central nervous system & Hemimegalencephaly \\
& Pachygyria \\
& Ventriculomegaly \\
& Mental retardation \\
& Seizures \\
& Ophthalmological \\
Other anomalies & Cardiac \\
& Urinary \\
\hline
\end{tabular}

Afflicted patients present with a roughly linear epidermal nevus following the lines of Blaschko, which represent the pattern of migration of ectodermal skin cells during embryogenesis. The phenomenon of the blaschkoid disposition, a recognized feature of cutaneous genetic mosaicism, supports the current idea that the multiorganic manifestations of ENS are due to genetic mosaicism [3].

Neurological manifestations include mental retardation and seizures [2]. The reported brain involvement in ENS is usually supratentorial, in most cases consisting of hemimegalencephaly, pachygyria and/or ventriculomegaly. Nevertheless, Abdelhalim et al. [4] have reported a severe ENS case with infratentorial anomalies along with major supratentorial structural changes. However, our patient presented only infratentorial anomalies. To our knowledge, this finding has not been previously reported. Another interesting feature of our patient is the finding of diffuse, but isolated, cerebellar dysplasia with no cerebral abnormalities, yet another highly unusual finding [5].

Dysplasia refers to the disorganized development of the cerebellum, as expressed by abnormal foliation and fissuration and/or the presence of gray matter heterotopias [6]. Several pathological mechanisms can cause cerebellar dysplasia [5,7], including infections, alcohol, radiation and genetic disorders. The latter may account for the cerebellar anomalies found in ENS and other neurocutaneous syndromes such as the hypomelanosis of Ito [8], in which an underlying genetic mosaicism is found. Accordingly, alterations in the genes involved in the various stages of cerebellar development-namely, the genes regulating the migration of Purkinje cells and, in particular, the prolif- eration and migration of granular cells-are presumed to be responsible for the cerebellar anomalies. As granular cells are the most abundant neurons in the cerebellum, their developmental course is thought to determine the size and patterns of the folia and fissures.

In addition to these changes, other systems may also be affected. Musculoskeletal changes are often found, with hemicorporal segmental hypertrophy (most commonly in the head or limbs) being a typical ENS feature. Ophthalmological problems are another common finding, whereas cardiac and urinary tract abnormalities are only occasionally reported.

To adequately evaluate the multisystemic involvement of the ENS, the use of imaging techniques is required. Neuroimaging modalities, especially MRI, play an important role as they provide better anatomical detail than other techniques, particularly when infratentorial structures are involved. For this reason, we believe that the increased use of MRI may well reveal further cerebellar abnormalities in ENS patients.

\section{Conflicts of interests}

None.

\section{References}

[1] Solomon LM, Fretzin DF, Dewald RL. The epidermal nevus syndrome. Arch Dermatol 1968;97:273-5.

[2] Pavone I, Curatolo P, Rizzo R, et al. Epidermal nevus syndrome: a neurologic variant with hemimegalencephaly, gyral malformation, mental retardation, seizures and facial hemihypertrophy. Neurology 1991;41:266-71.

[3] Sugarman JL. Epidermal nevus syndromes. Semin Cutan Med Surg 2004;23:145-57.

[4] Abdelhalim AN, Moritani T, Richfield E, et al. Epidermal nevus syndrome: megalencephaly with bihemispheric and cerebellar involvement: imaging and neuropathologic correlation. J Comput Assist Tomogr 2003;27:534-7.

[5] Soto-Ares G, Deries B, Delmaire C, et al. Dysplasie du cortex cérébelleux: aspects en IRM et signification. J Radiol 2004;85:729-40.

[6] Patel S, Barkovich AJ. Analysis and classification of cerebellar malformations. Am J Neuroradiol 2002;23:1074-87.

[7] Demaerel P. Abnormalities of cerebellar foliation and fissuration: classification, neurogenetics and clinicoradiological correlations. Neuroradiology 2002;44:639-46.

[8] Cianfoni A, Wintermark M, Piludu F, et al. Morphological and functional MR imaging of Lhermitte-Duclos disease with pathology correlate. J Neuroradiol 2008;35:297-300. 